\title{
Psychological distress and streamlined BreastScreen follow-up assessment versus standard assessment
}

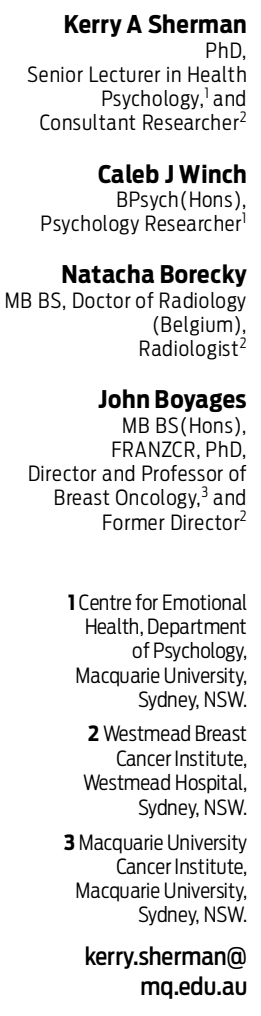

MJA 2013; 199: 599-603 doi: 10.5694/mjal3.10112 ustralian breast screening adheres to National Accreditation Standards to maximise cancer detection and minimise medical and psychological burdens associated with participation. ${ }^{1}$ However, by May 2007 the waiting period between an abnormal screening mammogram and follow-up assessment appointment at a large metropolitan program (BreastScreen NSW Sydney West [BSSW]) exceeded the required 28 days. In response, the Westmead Breast Cancer Institute (BCI), which coordinated BSSW, implemented a StepDown assessment protocol to streamline low-risk women to a mammogram-only clinic that completed assessments rapidly.

Attending a breast assessment clinic after a suspicious screening mammogram is associated with significant psychological burden, even after cancer is ruled out. ${ }^{2-4} \mathrm{~A}$ metaanalysis reported that although women with these false-positive screening mammograms do not typically experience generalised anxiety or depression, breast cancer-specific distress is heightened compared with women not requiring breast assessment. ${ }^{2}$ Although this distress usually diminishes substantially within weeks, it may remain somewhat elevated for years. ${ }^{3}$ Further, evidence from BreastScreen Western Australia ${ }^{5}$ and a meta-analysis of international programs ${ }^{4}$ show that false-positive results may discourage future screening. Consistent with an emphasis on the wellbeing of women screened, ${ }^{1}$ it is important to ensure that when implementing changes to standard protocol (ie, the StepDown approach adopted by BSSW), psychological burdens associated with attending an assessment clinic do not increase. Although the StepDown clinic was

\begin{abstract}
Objectives: To establish whether altered protocol characteristics of streamlined StepDown breast assessment clinics heightened or reduced the psychological distress of women in attendance compared with standard assessment. Willingness to attend future screening was also compared between the assessment groups.

Design: Observational, prospective study of women attending either a mammogram-only StepDown or a standard breast assessment clinic. Women completed questionnaires on the day of assessment and 1 month later.
\end{abstract}

Participants and setting: Women attending StepDown (136 women) or standard assessment clinics ( 148 women) at a BreastScreen centre between 10 November 2009 and 7 August 2010.

Main outcome measures: Breast cancer worries; positive and negative psychological consequences of assessment (Psychological Consequences Questionnaire); breast cancer-related intrusion and avoidance (Impact of Event Scale); and willingness to attend, and uneasiness about, future screening.

Results: At 1-month follow-up, no group differences were evident between those attending standard and StepDown clinics on breast cancer worries $(P=0.44)$, positive $(P=0.88)$ and negative $(P=0.65)$ consequences, intrusion $(P=0.64)$, and avoidance $(P=0.87)$. Willingness to return for future mammograms was high, and did not differ between groups $(P=0.16)$, although higher levels of unease were associated with lessened willingness to rescreen $(P=0.04)$.

Conclusions: There was no evidence that attending streamlined StepDown assessments had different outcomes in terms of distress than attending standard assessment clinics for women with a BreastScreen-detected abnormality. However, unease about attending future screening was generally associated with less willingness to do so in both groups; thus, there is a role for psycho-educational intervention to address these concerns.

not implemented specifically to address psychological burden, certain modified protocols have the potential to influence distress outcomes. The StepDown approach minimises waiting times between screening and assessment and conducts mammogram-only intervention at assessment. As longer waiting times and more invasive intervention are linked with increased distress, ${ }^{3}$ it is possible that the StepDown protocol will reduce distress compared with standard assessment. Past studies have shown that same-day assessment reduces distress compared with standard recall protocol, ${ }^{6,7}$ but it is unknown whether this benefit extends to the StepDown protocol because assessment is not on the same day. Indeed, qualitative research indicates that being rapidly recalled for assessment may in fact exacerbate distress, as women may worry that swift recall is indicative of malignancy; ${ }^{8}$ hence, the streamlined nature of StepDown assessments may actually heighten distress.

Accordingly, this prospective study aimed to compare levels of psychological distress among women attending StepDown versus standard assessment clinics, and who were found not to have cancer. Literature suggested that StepDown clinics could either reduce or heighten distress, so the study was designed to detect a difference in either direction. Additionally, factors associated with willingness to attend future screening were examined across both assessment clinic groups. 
1 Demographic details at baseline of 284 women attending StepDown or standard assessment clinics at a BreastScreen centre

\begin{tabular}{|c|c|c|c|c|}
\hline \multirow[b]{2}{*}{ Variable* } & \multicolumn{3}{|c|}{ Assessment clinic group } & \multirow[b]{2}{*}{ Total } \\
\hline & StepDown & Standard & $P$ & \\
\hline Mean age in years $(95 \% \mathrm{Cl}) ; n=283$ & $\begin{array}{c}59.1 \\
(58.1-60.2)\end{array}$ & $\begin{array}{c}56.6 \\
(55.4-57.8)\end{array}$ & 0.002 & $\begin{array}{c}57.8 \\
(57.0-58.6)\end{array}$ \\
\hline \multicolumn{5}{|l|}{ Country of origin } \\
\hline$n$ & 135 & 148 & & 283 \\
\hline Australia or New Zealand & $84(62.2 \%)$ & $109(73.6 \%)$ & & $193(68.2 \%)$ \\
\hline Western Europe & $23(17.0 \%)$ & $15(10.1 \%)$ & & $38(13.4 \%)$ \\
\hline Asia & $19(14.1 \%)$ & $12(8.1 \%)$ & & $31(11.0 \%)$ \\
\hline All other & $9(6.7 \%)$ & $12(8.1 \%)$ & 0.10 & $21(7.4 \%)$ \\
\hline \multicolumn{5}{|l|}{ Marital status } \\
\hline$n$ & 135 & 146 & & 281 \\
\hline Single, never married & $5(3.7 \%)$ & $9(6.2 \%)$ & & $14(5.0 \%)$ \\
\hline Married or living with partner & $103(76.3 \%)$ & $109(74.7 \%)$ & & $212(75.4 \%)$ \\
\hline Separated or widowed & $27(20.0 \%)$ & $28(19.2 \%)$ & 0.64 & $55(19.6 \%)$ \\
\hline \multicolumn{5}{|l|}{ Education level } \\
\hline$n$ & 135 & 145 & & 280 \\
\hline Up to year 10 & $57(42.2 \%)$ & $58(40.0 \%)$ & & 115 (41.1\%) \\
\hline Higher School Certificate & $15(11.1 \%)$ & $23(15.9 \%)$ & & $38(13.6 \%)$ \\
\hline Vocational or TAFE & $21(15.6 \%)$ & $28(19.3 \%)$ & & $49(17.5 \%)$ \\
\hline Began university & $42(31.1 \%)$ & $36(24.8 \%)$ & 0.42 & $78(27.9 \%)$ \\
\hline \multicolumn{5}{|l|}{ Blood relative with breast cancer } \\
\hline$n$ & 134 & 146 & & 280 \\
\hline Yes & $45(33.6 \%)$ & $49(33.6 \%)$ & & $94(33.6 \%)$ \\
\hline No & $70(52.2 \%)$ & $88(60.3 \%)$ & & $158(56.4 \%)$ \\
\hline Don't know & $19(14.2 \%)$ & $9(6.2 \%)$ & 0.07 & $28(10.0 \%)$ \\
\hline \multicolumn{5}{|l|}{ Previous breast cancer diagnosis } \\
\hline$n$ & 135 & 147 & & 282 \\
\hline Yes & $2(1.5 \%)$ & 0 & & $2(0.7 \%)$ \\
\hline No & $133(98.5 \%)$ & $147(100 \%)$ & na & $280(99.3 \%)$ \\
\hline $\begin{array}{l}\text { Mean days waited to be informed of } \\
\text { need for assessment }(95 \% \mathrm{Cl}) \\
n=260\end{array}$ & $\begin{array}{c}17.0 \\
(15.6-18.5)\end{array}$ & $\begin{array}{c}17.9 \\
(16.5-19.3)\end{array}$ & 0.41 & $\begin{array}{c}17.5 \\
(16.5-18.5)\end{array}$ \\
\hline $\begin{array}{l}\text { Mean no. of previous routine } \\
\text { mammograms }(95 \% \mathrm{Cl}) ; n=260\end{array}$ & $\begin{array}{c}6.7 \\
(5.8-7.5)\end{array}$ & $\begin{array}{c}4.6 \\
(3.8-5.4)\end{array}$ & 0.001 & $\begin{array}{c}5.6 \\
(5.0-6.2)\end{array}$ \\
\hline \multicolumn{5}{|c|}{ Recalled for further assessment at prior screening } \\
\hline$n$ & 133 & 144 & & 277 \\
\hline Yes & $47(35.3 \%)$ & $43(29.9 \%)$ & & 90 (32.5\%) \\
\hline No & $86(64.7 \%)$ & 101 (70.1\%) & 0.33 & $187(67.5 \%)$ \\
\hline
\end{tabular}

na $=$ not applicable. $* \chi^{2}$ test used for categorical variables, independent samples $t$ test used for numeric variables. $n$ varies across demographics because some women did not complete one or more items at baseline.

\section{Methods}

\section{Design and participants}

Recruitment occurred sequentially as women attended BSSW StepDown or standard assessment clinics at the BCI in Sydney from 10 November 2009 to 7 August 2010. The Sydney West Area Health Service, Westmead Campus, provided ethics approval. Eligibility criteria were age $\geqslant 40$ years and written English language competence. Women completed self-report questionnaires at baseline (in the clinic waiting room on their day of assessment) and 1 month later. Of 325 women approached by a researcher, 284 consented to participate and completed the baseline questionnaire $(87.4 \%$ recruitment). Ten women (3.1\%) declined because they were feeling too distressed. Follow-up questionnaires with return envelopes were posted 1 month later, accompanied by a telephone reminder. Dropout rates were similar across demographics and assessment group $\left(\chi^{2}=1.03 ; P=0.31\right)$. Four women with ongoing assessments and 16 women with malignant results were excluded from follow-up analysis to avoid confounding effects of assessment protocol with cancer diagnosis. Questionnaires were received from 198 of the remaining 264 women (75.0\% retention).

\section{Assessment clinic groups}

A nurse or counsellor recalled women by telephone using a script. All women were reassured that a cancer diagnosis was unlikely. Women recalled to StepDown assessment clinics were specifically informed that they met low-risk criteria and would undergo special mammograms only.

StepDown clinics began in December 2007. Eligibility for StepDown assessment reflected criteria consistent with low risk of cancer diagnosis: it was the woman's second or subsequent screen, abnormalities were graded at screening review as probably normal or benign, no indeterminate or suspicious calcification was present, and there was no dense breast tissue or lesions requiring ultrasound. The clinic involved special mammographic views only, batch-interpreted later by a breast surgeon and radiologist, who in most cases advised women to return to normal screening. But if cancer was not ruled out, women were reassigned to the standard clinic for further tests. About $13.4 \%$ of StepDown assessments in the 24 months before the psychosocial study resulted in reassignment.

Women not eligible for the StepDown clinic were allocated to standard assessment, entailing further mammograms and/or ultrasound, clinical examination by a doctor, and possible fine needle and/or core biopsy, until the nature of their abnormal mammogram was resolved.

\section{Measures}

Breast cancer-specific worry was measured using a four-item scale previously applied in mammographic screening. ${ }^{9}$ Women reported their current level of worry about breast cancer $(0=$ not at all to $4=$ almost all the time), the impact of worry on their mood and daily activities, and their anxiety about the results of future mammograms $(0=$ not at all to $3=\mathrm{a}$ lot). Impact items were summed 
2 Analyses of covariance for differences in psychosocial outcomes between the StepDown and standard clinics at baseline and 1-month follow-up.

\begin{tabular}{|c|c|c|c|c|c|c|c|}
\hline \multirow[b]{2}{*}{ Dependent variable } & \multicolumn{2}{|c|}{ Mean $(95 \% \mathrm{Cl})$ at baseline } & \multicolumn{2}{|c|}{$\begin{array}{c}\text { Adjusted mean* }(95 \% \mathrm{Cl}) \text { at } \\
\text { l-month follow-up }\end{array}$} & \multicolumn{3}{|c|}{$\begin{array}{l}\text { Effect of clinic group at } \\
\text { l-month follow-up }\end{array}$} \\
\hline & $\begin{array}{l}\text { StepDown } \\
(n=136)\end{array}$ & $\begin{array}{l}\text { Standard } \\
(n=148)\end{array}$ & $\begin{array}{l}\text { StepDown } \\
(n=105)\end{array}$ & $\begin{array}{l}\text { Standard } \\
(n=93)\end{array}$ & $n^{\dagger}$ & $F$ & $P$ \\
\hline \multicolumn{8}{|l|}{ Worry about breast cancer } \\
\hline Current level of worry & $1.59(1.43-1.75)$ & $1.58(1.44-1.72)$ & $1.57(1.40-1.73)$ & $1.66(1.49-1.84)$ & 178 & 0.60 & 0.44 \\
\hline Negative impact of worry & $1.27(1.02-1.53)$ & $1.18(0.96-1.40)$ & $0.75(0.51-0.98)$ & $0.91(0.66-1.16)$ & 177 & 0.82 & 0.37 \\
\hline $\begin{array}{l}\text { Anxiety about future } \\
\text { mammographic results }\end{array}$ & $1.52(1.38-1.66)$ & $1.41(1.28-1.55)$ & $1.18(1.01-1.34)$ & $1.20(1.02-1.37)$ & 177 & 0.03 & 0.86 \\
\hline \multicolumn{8}{|l|}{ Psychological consequences } \\
\hline Negative & $7.75(6.40-9.11)$ & $7.22(5.95-8.48)$ & $4.99(3.68-6.29)$ & $4.54(3.20-5.88)$ & 173 & 0.21 & 0.65 \\
\hline Positive & - & - & $11.97(9.78-14.14)$ & $12.21(9.96-14.45)$ & 176 & 0.02 & 0.88 \\
\hline \multicolumn{8}{|l|}{ Impact of the recall event } \\
\hline IES intrusion & $7.98(6.69-9.26)$ & $7.46(6.19-8.73)$ & $5.99(4.47-7.51)$ & $5.45(3.85-7.04)$ & 174 & 0.23 & 0.64 \\
\hline IES avoidance & $11.57(9.81-13.33)$ & $11.71(10.08-13.35)$ & $8.16(6.16-10.17)$ & $8.41(6.32-10.51)$ & 173 & 0.03 & 0.87 \\
\hline Future screening intentions & $4.64(4.50-4.78)$ & $4.54(4.39-4.69)$ & $4.76(4.32-4.88)$ & $4.62(4.49-4.76)$ & 176 & 2.01 & 0.16 \\
\hline $\begin{array}{l}\text { Uneasiness about attending } \\
\text { future screening }\end{array}$ & $2.16(1.93-2.38)$ & $2.16(1.95-2.37)$ & $2.24(2.01-2.48)$ & $2.24(1.99-2.48)$ & 175 & $<0.01$ & 0.98 \\
\hline
\end{tabular}

(range, 0-6; Cronbach $\alpha=0.78$ ). Higher scores indicated more negative impact.

The reliable and valid Psychological Consequences Questionnaire (PCQ $)^{10}$ was used to measure the emotional, social and physical consequences of breast cancer screening. Twelve items assessed negative consequences $(0=$ not at all to $3=$ quite a lot of the time). Higher summed scores indicated more negative consequences (range, 0-36; Cronbach $\alpha=$ 0.92). The remaining 10 items assessed positive consequences. Higher summed scores indicated more positive consequences (range, 0-30; Cronbach $\alpha=0.95)$. As recommended, the positive consequences subscale was presented at follow-up only.

The Impact of Event Scale (IES) ${ }^{11}$ was used to measure psychological distress caused by breast assessment. Women rated, in relation to their clinic visit, the frequency of intrusive (seven items) and avoidant (eight items) thoughts and feelings about breast cancer. Responses $(0=$ not at all, $1=$ rarely, $3=$ sometimes, $5=$ often) were summed to produce intrusion (range, 0-35; Cronbach $\alpha=$ 0.87 ) and avoidance (range, 0-40; Cronbach $\alpha=0.88$ ) subscales. Higher scores indicated more symptoms of distress.
Two items related to future screening. Women rated how likely they were to attend future routine breast screening appointments ( 1 = not likely at all to $5=$ extremely likely), and how uneasy they felt about attending future breast screening $(1=$ not at all to $5=$ extremely uneasy).

Age, country of origin, marital status, education and medical history were documented only at baseline. All other measures were taken at both time points.

\section{Power analysis}

The study was designed to detect a small-to-moderate difference in distress between groups, corresponding to a standardised difference $d=0.40$ units of SD. The simplest test of this difference, an independent samples $t$ test, could detect a standardised difference $d=0.39$ with 0.80 power at $P \leqslant 0.05$ (two-tailed) with $N=200$.

\section{Data analysis}

Descriptive statistics were computed for baseline demographic and medical history variables. Potential confounding variables were identified by comparing StepDown clinic attendees and standard assessment attendees on baseline demographic and medical variables using $t$ tests and $\chi^{2}$ procedures. The significance criterion $P \leqslant 0.10$ was used to identify con- founders because clinic assignment was non-random in the naturalistic context of the screening service. This criterion confers conservatism by identifying potential confounders, even trending towards an a-priori difference between groups. Such variables were included as covariates in subsequent analyses, which were undertaken at the conventional significance criterion of $P \leqslant 0.05$. Two-way imputation was applied to incomplete questionnaires for subscales with up to one-third items missing. ${ }^{12}$ This is superior to deleting participants with missing data ${ }^{13}$ or imputing the participant's mean on other items. ${ }^{14}$ Analyses of covariance (ANCOVAs) were then conducted, controlling for baseline values of the outcome, to identify differences in psychosocial outcomes between the StepDown and standard clinics at 1-month follow-up. To identify predictors of willingness to attend future routine breast screenings, the psychosocial variables were entered into a multiple regression.

\section{Results}

Demographic and medical variables are summarised in Box 1. Standard and StepDown assessment groups differed on age, country of birth, number of previous mammograms, and family history of breast cancer; 
3 Multiple regression model predicting willingness to return to screening in 166 women attending StepDown or standard assessment clinics

\begin{tabular}{|c|c|c|c|}
\hline \multirow[b]{2}{*}{ Predictor } & \multicolumn{3}{|c|}{$\begin{array}{l}\text { Likelihood of attending future routine } \\
\text { breast screening appointments }\end{array}$} \\
\hline & $b *$ & $\begin{array}{l}\text { Standard } \\
\text { error of } b\end{array}$ & $P$ \\
\hline Likelihood at baseline & 0.56 & 0.07 & $<0.001$ \\
\hline \multicolumn{4}{|l|}{ Assessment clinic type } \\
\hline Standard ${ }^{\dagger}$ & - & - & - \\
\hline StepDown & 0.11 & 0.10 & 0.25 \\
\hline Age & 0.01 & 0.01 & 0.20 \\
\hline \multicolumn{4}{|l|}{ Country of birth } \\
\hline Australia or New Zealand ${ }^{\dagger}$ & - & - & - \\
\hline Western Europe & 0.02 & 0.16 & 0.88 \\
\hline Asia & 0.01 & 0.16 & 0.97 \\
\hline All others & 0.12 & 0.28 & 0.66 \\
\hline Number of prior routine mammograms & -0.02 & 0.01 & 0.08 \\
\hline \multicolumn{4}{|l|}{ Blood relatives with breast cancer } \\
\hline $\mathrm{No}^{+}$ & - & - & - \\
\hline Yes & 0.07 & 0.11 & 0.56 \\
\hline Unknown & 0.13 & 0.17 & 0.46 \\
\hline \multicolumn{4}{|l|}{ Worry about breast cancer } \\
\hline Current level of worry & 0.11 & 0.07 & 0.15 \\
\hline Negative impact of worry & -0.09 & 0.06 & 0.10 \\
\hline Anxiety about future mammographic results & 0.07 & 0.09 & 0.43 \\
\hline \multicolumn{4}{|l|}{ Psychological consequences } \\
\hline Negative & 0.01 & 0.01 & 0.52 \\
\hline Positive & $<0.01$ & 0.01 & 0.95 \\
\hline \multicolumn{4}{|l|}{ Impact of the recall event } \\
\hline IES intrusion & -0.01 & 0.01 & 0.56 \\
\hline IES avoidance & 0.01 & 0.01 & 0.17 \\
\hline Uneasiness about attending future screening & -0.10 & 0.05 & 0.04 \\
\hline
\end{tabular}

IES = Impact of Event Scale. $" * b$ is the predicted change in willingness to attend future screening (range, $1=$ not likely at all to $5=$ extremely likely) associated with a 1-unit increase in the corresponding independent variable. All psychological outcomes are measured at follow-up, excep where specified. † Reference group.

these variables were treated as covariates in further analyses, and adjusted means are reported.

Mean baseline psychosocial outcomes (unadjusted) for the standard and StepDown assessment groups are displayed in Box 2. No baseline outcomes differed by clinic group, controlling for the covariates (ANCOVA: all $F$ values $<2.43$, all $P$ values $>0.12$ ).

Levels of breast cancer worry, perceived negative consequences, and distress (intrusion) were low at baseline and follow-up for both groups. However, baseline and follow-up avoidance-related distress was moderately high (Box 2). Controlling for covariates and baseline values of the psychosocial outcomes, ANCOVAs found no significant differences in psychosocial outcomes between the assessment groups at follow-up (Box 2).
At follow-up, participants across both groups reported a high likelihood of attending future routine screening (mean, 4.72; 95\% CI, 4.624.82). Multiple regression showed that for the combined sample this likelihood was most strongly related to prior self-rated likelihood at baseline. However, a lower likelihood of rescreening was associated with feeling uneasy about rescreening, irrespective of baseline likelihood or assessment clinic type (Box 3). Nevertheless, this should be interpreted within the overall findings, as $65.2 \%$ of women reported little or no unease about future screening.

\section{Discussion}

This study compared psychosocial responses of women recalled to attend either a StepDown or standard breast assessment clinic at BSSW. Overall, women reported moderateto-low distress associated with their false-positive breast screen result 1 month after attending assessment, with no differences between the two clinic types.

In both assessment groups, women had low worry about being diagnosed with breast cancer, low negative impact of the worry, and low anxiety about future mammographic results. These outcomes are comparable with the 6-month follow-up of women who underwent breast assessment in North America. ${ }^{9}$ Perceived negative psychological consequences of being recalled were low in both assessment groups and consistent with studies using the PCQ 2 weeks after assessment (Australia) ${ }^{15}$ and 6 months after assessment (Sweden). ${ }^{16}$ Moreover, women in both assessment groups perceived moderate positive consequences of the experience, reflecting similar findings among women in the United States 1 month after their false-positive result. ${ }^{17}$

Women attending BSSW clinics reported low, subclinical levels of cancer-specific intrusive thoughts. This level of intrusion (mean, 5.99) was slightly lower than that reported by French women after screening mammograms (mean, 8.50) as part of high-risk surveillance. ${ }^{18}$ However, women in both BSSW groups experienced moderate symptoms of avoidant-related distress, a level comparable to recently bereaved individuals, $^{19}$ at baseline and follow-up. Although this distress was not directly related to future screening intentions, another study linked higher IES distress to non-adherence to mammographic screening. ${ }^{20}$ Interventions that address concerns and identify barriers (eg, problem-solving training) have increased breast self-examination in women with high IES scores; ${ }^{21}$ similar efforts may improve rescreening where a false-positive mammogram results in prolonged distress.

A key finding of this study is that women attending StepDown clinics did not experience significantly different levels of psychological burden than women attending the standard assessment clinic. This is not due to insufficient statistical power, as the 
study was designed to detect a smallto-moderate difference, $d=0.40$, whereas the group means at followup (Box 2) suggest small-to-nil differences ranging from $d=0.10$ (negative impact of breast cancer worry) to $d=$ 0.02 (positive psychological consequences). However, as the streamlined StepDown protocol entails fewer diagnostic interventions and a rapid recall time frame, there was potential for women to be less distressed than women attending standard clinics. That this did not occur highlights the challenge of reducing distress associated with recall, but is consistent with evidence that assessment is stressful for women regardless of the diagnostic investigations. ${ }^{3}$ Some women declined to enter the present study because they were too distressed, meaning distress levels may be slightly underestimated. Overall, though, the psychological burden of assessment at BSSW was comparable to other studies using the same measures and pre-post designs. ${ }^{9,15-18}$

Women in both assessment clinics reported being very likely to return to future screening. Further, two-thirds of women reported little or no uneasiness about rescreening. However, the more uneasy women felt about future screening, the lower their selfreported likelihood of rescreening. Such unease may discourage reattendance for the third of the sample who reported being moderately, very or extremely uneasy about rescreening. Unease showed low-tomoderate associations with all measures of psychological burden, suggesting that unease may reflect a range of concerns raised by false-positive breast assessment. Supporting this, research examining previously assessed women in the United Kingdom just before their next routine screen found that worse PCQ scores were associated with more worry about the test results, doubts about test accuracy and not having a person to contact at the screening service. ${ }^{22}$ Further, when considering whether to rescreen, these women reported being worried by magazine, newspaper and television accounts of screening, but were also influenced by health posters or leaflets and their GP's attitude. Thus, a woman's unease about her breast assessment experience can be reduced through support from the breast clinic and her GP. ${ }^{1,15,23}$

This is the first study to investigate distress associated with different assessment protocols in Australia. While the self-reported nature of these data needs to be noted, they represent women attending breast assessment well: there were high rates of recruitment $(87 \%)$ and retention $(75 \%)$. In conclusion, a streamlined StepDown breast assessment clinic was not different in terms of distress compared with standard assessment. It allowed BSSW to rapidly obtain extra mammographic views, separately from the standard clinic and without on-site medical review, and consequently to meet accreditation standards. Australian breast screening programs should continue to investigate psychological distress associated with changes in program protocols.

Acknowledgements: We would like to acknowledge NSW Health, from which the Westmead Breast Cancer Institute receives funding. We thank Sarah Ellis and Claire Gore for assisting with recruitment, and Matthew Beacom for contributing to manuscript preparation.

Competing interests: No relevant disclosures.

Received 24 Jan 2013, accepted 6 Aug 2013.

1 BreastScreen Australia Quality Improvement Program. BreastScreen Australia National Accreditation Standards. 2010. http:// www.cancerscreening.gov.au/internet/ screening/publishing.nsf/Content/braccreditation/\$File/standards.pdf (accessed Nov 2012).

2 Salz T, Richman AR, Brewer NT. Meta-analyses of the effect of false-positive mammograms on generic and specific psychosocial outcomes. Psychooncology 2010; 19: 1026-1034.

3 Brett J, Bankhead C, Henderson B, et al. The psychological impact of mammographic screening. A systematic review. Psychooncology 2005; 14: 917-938.

4 Brewer NT, Salz T, Lillie SE. Systematic review: the long-term effects of false-positive mammograms. Ann Intern Med 2007; 146: 502-510.

5 Sim MJ, Siva SP, Ramli IS, et al. Effect of falsepositive screening mammograms on rescreening in Western Australia. Med J Aust 2012; 196: 693-695.

6 Barton MB, Morley DS, Moore S, et al. Decreasing women's anxieties after abnormal mammograms: a controlled trial. J Natl Cancer Inst 2004; 96: 529-538.

7 Lindfors KK, O'Connor J, Parker RA. False-positive screening mammograms: effect of immediate versus later work-up on patient stress. Radiology 2001; 218: 247-253.

8 Solbjør M, Forsmo S, Skolbekken JA, Sætnan AR Experiences of recall after mammography screening-a qualitative study. Health Care Women Int 2011; 32: 1009-1027.

9 Lerman C, Trock B, Rimer BK, et al. Psychological side effects of breast cancer screening. Health Psychol 1991; 10: 259-267.

10 Cockburn J, De Luise T, Hurley S, Clover K. Development and validation of the PCQ: a questionnaire to measure the psychological consequences of screening mammography. Soc Sci Med 1992; 34: 1129-1134.

11 Horowitz M, Wilner N, Alvarez W. Impact of Event Scale: a measure of subjective stress. Psychosom Med 1979; 41: 209-218.

12 van Ginkel JR, van der Ark LA. SPSS syntax for missing value imputation in test and questionnaire data. Applied Psychological Measurement 2005; 29: 152-153. doi: 10.1177/ 0146621603260688.

13 Van der Ark LA, Sijtsma K. The effect of missing data imputation on Mokken Scale Analysis. In: Van der Ark LA, Croon MA, Sijtsma K, editors. New developments in categorical data analysis for the social and behavioral sciences. Mahwah, NJ: Erlbaum, 2005.

14 Sijtsma K, van der Ark LA. Investigation and treatment of missing item scores in test and questionnaire data. Multivariate Behavioural Research 2003; 38: 505-528. doi: 10.1207/ s15327906mbr3804_4.

15 Bowland L, Cockburn J, Cawson J, et al. Counselling interventions to address the psychological consequences of screening mammography: a randomised trial. Patient Educ Couns 2003; 49: 189-198.

16 Olsson P, Armelius K, Nordahl G, et al. Women with false positive screening mammograms: how do they cope? J Med Screen 1999; 6: 89-93.

17 Tyndel S, Austoker J, Henderson BJ, et al. What is the psychological impact of mammographic screening on younger women with a family history of breast cancer? Findings from a prospective cohort study by the PIMMS Management Group. J Clin Oncol 2007; 25: 3823-3830.

18 Brédart A, Kop JL, Fall M, et al. Anxiety and specific distress in women at intermediate and high risk of breast cancer before and after surveillance by magnetic resonance imaging and mammography versus standard mammography. Psychooncology 2011; 21: 1185-1194.

19 Fischer J, Corcoran K. Measures for clinical practice: a sourcebook. Vol. 2: adults. 3rd ed. New York: The Free Press, 1994.

20 Lerman C, Daly M, Sands C, et al. Mammography adherence and psychological distress among women at risk for breast cancer. J Natl Cancer Inst 1993; 85: 1074-1080.

21 Audrain J, Rimer B, Cella D, et al. The impact of a brief coping skills intervention on adherence to breast self-examination among first-degree relatives of newly diagnosed breast cancer patients. Psychooncology 1999; 8: 220-229.

22 Brett J, Austoker J. Women who are recalled for further investigation for breast screening: psychological consequences 3 years after recall and factors affecting re-attendance. $J$ Public Health Med 2001; 23: 292-300.

23 Ong G, Austoker J. Recalling women for further investigation of breast screening: women's experiences at the clinic and afterwards. J Public Health Med 1997; 19: 29-36. 